\title{
SUSY and Dark Matter Results from ATLAS
}

\author{
H. Sandaker* on behalf of the ATLAS collaboration \\ Department of Physics and Technology, University of Bergen, \\ 5007 Bergen, Norway \\ *E-mail: Heidi.Sandaker@ift.uib.no \\ www.uib.no
}

\begin{abstract}
New results from the LHC are increasingly probing the borders of the Standard Model of particle physics. Some of the most attractive scenarios for new physics are supersymmetric models. In addition to solving some of the shortcomings of the Standard Model (e.g. the hierarchy problem, gauge coupling unification) they also provide a suitable Dark Matter candidate, which could be produced at the LHC. We present the latest searches for Supersymmetry in events with high-energy final states and large missing transverse momentum using $4.7 \mathrm{fb}^{-1}$ of proton-proton collisions at $\sqrt{s}=7 \mathrm{TeV}$ as recorded by the ATLAS detector at the Large Hadron Collider. The data is interpreted in models where the Dark Matter candidate is dominantly produced in cascade decays of heavier unstable supersymmetric particles together with high- $p_{T}$ Standard Model particles. We also present more model-independent searches for one single highly energetic jet or photon together with large amount of missing transverse energy, showing the first results for a mono-jet analysis. Such signals are highly relevant for Dark Matter studies and assume that the DM candidates are pair-produced at the LHC and that all other particles are too heavy to be produced directly. So far no excess above the Standard Model background expectation is observed.
\end{abstract}

Keywords: ATLAS, LHC, SUSY, Dark Matter, Mono-jet, Mono-photon

\section{Introduction}

The ATLAS experiment, ${ }^{1}$ a general purpose detector situated at one of the four collision points of the Large Hadron Collider (LHC), has collected data at unprecedented energies since the start of operation of LHC. In 2011, ATLAS collected 4.7 $\mathrm{fb}^{-1}$ of data at $\sqrt{s}=7 \mathrm{TeV}$. We present supersymmetric and mono-jet/mono-photon searches performed with the 2011 ATLAS data and their Dark Matter interpretation.

\section{Supersymmetric searches}

Supersymmetry (SUSY) ${ }^{2}$ is one of the most promising theories beyond the Standard Model (SM), proposing solutions for some of the most important open questions of the Standard Model such as the hierarchy problem, unification of the gauge couplings. It also provides a natural cancellation of corrections to the Higgs mass. In addition, this theory provides suitable Dark Matter candidates compatible with astrophysics and astro-particle physics observations such as the $\mathrm{WMAP}^{3}$ measurements of the cosmic microwave background. For these reasons the search for SUSY is one the most important goals of the ATLAS experiment with about 30 different analyses performed in 2011. These provide new exclusion limits for various models, ${ }^{2}$ such as minimal Supergravity (mSUGRA), Gauge-Mediated Supersymmetry Breaking (GMSB), phenomenological minimal Supersymmetric Standard Model 
(pMSSM) as well as in models where R-parity (quantum number) is violated.

If R-parity is conserved, all SUSY particles at the LHC will be pair produced. Production of squarks and gluinos dominates if these particles are kinematically accessible, while direct production of charginos, neutralinos and sleptons occurs via electroweak processes leading to much smaller cross sections. The produced supersymmetric particles will then cascade decay (fig. 1) into lighter particles, always ending with the lightest SUSY particle (LSP). The final state: nJets $+E_{T}^{\text {miss }}+$ $X$, contains a number of very energetic jets, the LSP seen in ATLAS as missing transverse energy and an additional particle $\mathrm{X}$ (e.g. $0,1,2$ or multi-leptons, 1 or 2 taus, a b-jet) which are are used to distinguish between the different final states.

In ATLAS data, cuts on jets and $E_{T}^{m i s s}$ is applied to distinguish the signal from the SM background. Each model studied optimises sensitivity by applying cuts on additional discriminating variables. So far no statistically significant excess has been observed. In the absence of any discovery we set exclusion limits. A subset of the most important new exclusion limits presented in summer/autumn 2011 for the 2011 dataset and $4.7 \mathrm{fb}^{-1}$ are listed below together with an example of a typical exclusion limit at $95 \%$ CL:

0 lepton + 2-6 jets $+E_{T}^{\text {miss }}$ Squarks and gluinos of equal mass are excluded for masses below $1360 \mathrm{GeV}$ (mSUGRA/CMSSM). ${ }^{4}$

0 lepton + 6-9 jets $+E_{T}^{\text {miss }}$ Gluino masses smaller than $840 \mathrm{GeV}$ are excluded for large universal scalar mass $m_{0}$ (mSUGRA/CMSSM). ${ }^{5}$

1 lepton + jets $+E_{T}^{\text {miss }}$ Top squark masses between 230 and $440 \mathrm{GeV}$ are excluded for massless LSPs, and $\sim 400 \mathrm{GeV}$ for LSP masses up to $125 \mathrm{GeV} .^{6}$

2 lepton + jets $+E_{T}^{\text {miss }}$ Light scalar top quark masses below $130 \mathrm{GeV}$ are excluded for neutralino masses below $70 \mathrm{GeV}$ (very light stop). ${ }^{7}$

$\geqslant 1 \tau+$ jets $+E_{T}^{m i s s}$ Lower limits on the GMSB mass scale $\Lambda$ are set at $54 \mathrm{TeV}$ in the regions where the stau is the $\operatorname{NLSP}(\tan (\beta)>20)(\operatorname{GMSB}){ }^{8}$

Disappearing track + jets $+E_{T}^{\text {miss }}$ A chargino having a mass below 103 (85) $\mathrm{GeV}$ with a mass splitting of 160 (170) $\mathrm{MeV}$ is excluded (ASMB). ${ }^{9}$

3 b-jets +0 lepton + jets $+E_{T}^{\text {miss }}$ Exclusion limits for a variety of gluinomediated models with gluino masses up to $1 \mathrm{TeV}$ are excluded. ${ }^{10}$

\section{Model Independent searches}

In addition to model specific searches in ATLAS such as searches for SUSY particles, ATLAS also performs more model independent searches. These analyses assume that Weakly Intarctive Massive Particles (WIMPS) are pair produced at LHC and tagged by a jet or a photon. It is also assumed that all new particles mediating the interaction between WIMPs and the SM are too heavy to be produced directly and can thus be integrated out in an effective field theory. The typical signal in the LHC would be $E_{T}^{\text {miss }}$ as well as one jet or one photon. Theses two signatures has been used to study both the phenomenology of supersymmetry and extra dimensions.

The first results for the monojet analysis were published summer 2011. ${ }^{11}$ No 
excess has been found above the SM background and upper limits are set at 95\% CL on the visible cross section (from $1.92 \mathrm{pb}$ to $7 \mathrm{fb}$ in the various signal region) of any non-SM contribution. The results were also used to set limits on a mass suppression scale $M_{*}$ for pair production of WIMP dark matter particles. Within this approach the ATLAS limits can be converted to limits on WIMP-nucleon scattering and annihilation cross-sections (see fig. 1).

\section{LHC and Dark Matter}

The complementarity between direct, indirect and collider experiments is increasingly important. SUSY particle searches in ATLAS could find several Dark Matter candidates (e.g. neutralino, gravitino), but the model dependency makes it difficult to compare results directly with astroparticle physics results. It is possible, however, in some models, to make an estimate. For mSUGRA/CMSSM (fig. 1) the gluino and neutralino mass relation is $m_{\text {gluino }} \sim 6.4 m_{\chi}$ in the gaugino region, ${ }^{13}$ excluding neutralino masses $\sim 125 \mathrm{GeV}$ for gluino masses of $800 \mathrm{GeV}$ for this particular model.

ATLAS monojet and monophoton searches have the advantage that they can be directly compared with astroparticle experiments. In fig. 1 the recent ATLAS monojet limit shows that for WIMP masses below $10 \mathrm{GeV}$ for vector couplings and below $\sim 100 \mathrm{GeV}$ for axial-vector couplings the ATLAS limits are stronger than the FERMI-LAT ${ }^{12}$ limits.

If DM particles exists and are within the reach of the LHC, ATLAS could be able to detect suitable candidates and measure their masses and properties (e.g. decay chains, mass differences, signal strengths, mass scale) and also say something about the underlaying theory. The implication of the Higgs mass on different SUSY scenarios shows that if the newly discovered boson ${ }^{15}$ is a Higgs with mass of around $125 \mathrm{GeV}$ the mass scale for SUSY is moved upwards but quite possibly still within reach of the LHC. ${ }^{15}$

\section{Summary and conclusion}

LHC and ATLAS perform very well. By summer 2012, the first observation of a new boson was made, ${ }^{14}$ but no excess above the Standard Model background is observed for supersymmetric searches and ATLAS is excluding larger and larger regions of the SUSY parameter space. The model independent searches set new constraints on the WIMP-nucleon cross-section and $\gamma \gamma$ annihilation cross-section compatible with astroparticle experiments.

In 2012 the energy was increased to $8 \mathrm{TeV}$ and by July 2012 a record luminosity of $6.5 \mathrm{fb}^{-1}$ had been collected. With the new data of 2012, new results are imminent. Many of the most interesting theories for Dark Matter give large signals for the LHC. ATLAS data are not only compatible with astroparticle physics results but could also be the one of the first to discover of a Dark Matter candidate. 


\section{References}

1. ATLAS collaboration, ATLAS detector(2008 JINST 3 S08003)

2. S. P. Martin, A Supersymmetry primer hep-ph/9709356.

3. WMAP collaboration, E. Komatsu et al., Seven-year Wilkinson Microwave Anisotropy Probe (WMAP) observations: cosmological interpretation (Astrophys. J. Suppl. 192 (2011) 18)

4. ATLAS collaboration, Search for squarks and gluinos with the ATLAS detector in final states with jets and missing transverse momentum using $4.7 \mathrm{fb}^{-1}$ of $\sqrt{\mathrm{s}}=7 \mathrm{TeV}$ protonproton collision data (PRD 87 (2013) 012008)

5. ATLAS collaboration, Hunt for new phenomena using large jet multiplicities and missing transverse momentum with ATLAS in $4.7 \mathrm{fb}^{-1}$ of $\sqrt{s}=7$ TeV proton-proton collisions (JHEP 1207 (2012) 167)

6. ATLAS collaboration, Search for direct top squark pair production in final states with one isolated lepton, jets, and missing transverse momentum in sqrt(s) $=7 \mathrm{TeV} p p$ collisions using $4.7 \mathrm{fb}-1$ of ATLAS data (PRL 109 (2012) 211803)

7. ATLAS collaboration, Search for light scalar top quark pair production in final states with two leptons with the ATLAS detector in $\sqrt{s}=7$ TeV proton-proton collisions (EPJC (2012) 72 2237)

8. ATLAS collaboration, Search for Supersymmetry in Events with Large Missing Transverse Momentum, Jets, and at Least One Tau Lepton in 7 TeV Proton-Proton Collision Data with the ATLAS Detector (EPJC 72 (2012) 2215)

9. ATLAS collaboration, in Search for direct chargino production in anomaly-mediated supersymmetry breaking models based on a disappearing-track signature in pp collisions at $s=7$ TeV with the ATLAS detector(JHEP 01 (2013) 131)

10. ATLAS collaboration, Search for top and bottom squarks from gluino pair production in final states with missing transverse energy and at least three b-jets with the ATLAS detector (EPJC 72 (2012) 2174)

11. ATLAS collaboration, Search for dark matter candidates and large extra dimensions in events with a jet and missing transverse momentum with the ATLAS detector(JHEP
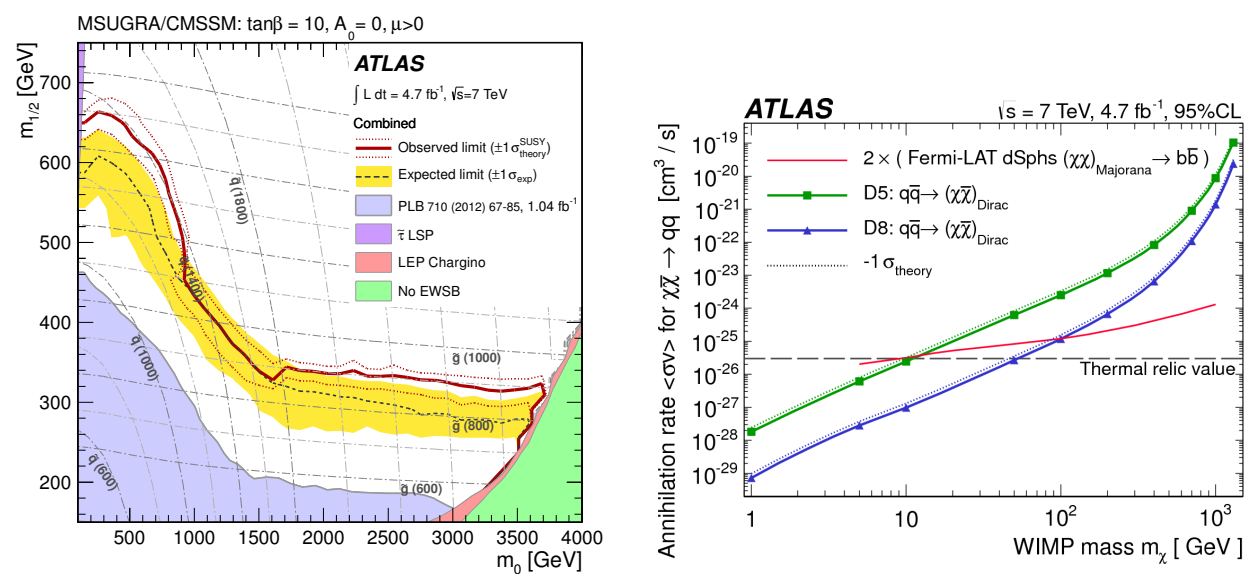

Fig. 1. (a) Supersymmetry exclusion limit for a search of a signature with zero leptons and 2-6 jets and missing transverse energy ${ }^{4}$ (b) Exclusion for finding a monojet signal and missing transverse energy compared to the FERMI-LAT limit. ${ }^{11}$ 
$04(2013) 75)$

12. Fermi-LAT collaboration, Constraining dark matter models from a combined analysis of Milky Way satellites with the Fermi Large Area Telescope (Phys. Rev. Lett. 107 (2011) 241302)

13. R. Arnowitt, A. Chamseddine and P. Nath, N=1 Supergravity (World Scientific, Singapore, 1984)

14. ATLAS collaboration, Observation of a new particle in the search for the Standard Model Higgs boson with the ATLAS detector at the LHC (Phys. Lett. B, Vol. 716, Issue 1(2012) 129)

15. O. Buchmueller et al. Higgs and Supersymmetry (Eur. Phys. J. C (2012) 72: 2020) 